\title{
Simultaneous X-ray and infrared variability in the quasar 3C273 - II. Confirmation of the correlation and X-ray lag
}

\author{
Ian $\mathrm{M}^{\mathrm{c}}$ Hardy, ${ }^{1 \star}$ Anthony Lawson, ${ }^{1} \dagger$ Andrew Newsam, ${ }^{1,2}$ Alan P. Marscher, ${ }^{3}$ \\ Andrei S. Sokolov, ${ }^{3}$ C. Megan Urry ${ }^{4}$ and Ann E. Wehrle ${ }^{5}$ \\ ${ }^{1}$ School of Physics and Astronomy, University of Southampton SO17 1BJ \\ ${ }^{2}$ Liverpool John Moores University, Astrophysics Research Institute, Birkenhead CH41 1LD \\ ${ }^{3}$ Institute for Astrophysical Research, 725 Commonwealth Avenue, Boston University, Boston, MA 02215, USA \\ ${ }^{4}$ Department of Physics, Yale University, PO Box 208121, New Haven, CT 06520-8121, USA \\ ${ }^{5}$ IPAC, Jet Propoulsion Laboratory and California Institute of Technology, Pasadena, CA 91125, USA
}

Accepted 2006 December 18. Received 2006 December 18; in original form 2006 September 17

\begin{abstract}
The X-ray emission from quasars, such as $3 \mathrm{C} 273$, is generally agreed to arise from Compton scattering of low-energy seed photons by relativistic electrons in a relativistic jet oriented close to the line of sight. However, there are a number of possible models for the origin of the seed photons. In Paper I ( $\mathrm{M}^{\mathrm{c}}$ Hardy et al.), we showed that the X-ray and infrared (IR) variability from 3C273 was highly correlated in 1997, with the IR flux leading the X-rays by $\sim 0.75 \pm$ $0.25 \mathrm{~d}$. The strong correlation, and lag, supports the synchrotron self-Compton (SSC) model, where the seed photons are synchrotron photons from the jet itself.

The previous correlation was based on one moderately well-sampled flare and another poorly sampled flare, so the possibility of chance correlated variability exists. Here, we report on further X-ray and IR observations of 3C273 which confirm the behaviour seen in Paper I. During a 2-week period of observations, we see a flare of amplitude $\sim 25$ per cent, lasting for $\sim 5 \mathrm{~d}$, showing a high correlation between IR and $\mathrm{X}$-ray variations, with the $\mathrm{X}$-rays lagging by $\sim 1.45 \pm 0.15 \mathrm{~d}$. These observations were not scheduled at any special time, implying that the same mechanism - almost certainly SSC - dominates the X-ray emission on most occasions and that the structure of the emission region is similar in most small flares.
\end{abstract}

Key words: galaxies: active - quasars: individual: 3C273 - X-rays: galaxies.

\section{INTRODUCTION}

The quasar $3 \mathrm{C} 273$ is a non-extreme, but very bright, example of the class of active galactic nuclei known as blazars. Blazars are characterized by high polarization (Valtaoja et al. 1990) and violent variability at optical wavelengths. In the case of $3 \mathrm{C} 273$, the blazar nature is more apparent at near-infrared than at optical wavelengths (Robson et al. 1993). Blazars contain a strong, relativistically flowing, non-thermally emitting jet, which features superluminal apparent motion of pc-scale radio components (e.g. Mantovani et al. 1999; Jorstad et al. 2001, 2005, and references therein). It is almost universally accepted that the radio through optical emission in such quasars is synchrotron emission from a relativistic jet oriented close to the line of sight. The smooth synchrotron spectrum does not generally extend to the X-ray emission, which is best explained by Compton scattering of seed photons by the relativistic electrons

^E-mail: imh@astro.soton.ac.uk

†Present address: QinetiQ, St Andrews Road, Malvern WR14 3PS. in the jet. 3C273 has been widely observed at X- and Gamma-ray energies (see e.g. Courvoisier 1998; Courvoisier et al. 2003), but the origin of the seed photons, particularly for the higher energies, remains one of the major questions in quasar physics.

As well as emission from the relativistic jet, 3C273 also contains a Seyfert-like nucleus which contributes unbeamed X-ray emission (e.g. Grandi \& Palumbo 2004), perhaps accounting for $\sim 20$ per cent of the total X-ray flux. The accretion disc surrounding the central black hole produces strong ultraviolet (UV) and optical emission (see e.g. Courvoisier et al. 2003), and infrared (IR) emission in 3C273 can arise from synchrotron radiation from the jet, free-free emission from dense clouds (Robson et al. 1986) on sub-pc-scales, or thermal emission from a hot dusty torus on a $\sim 10$ pc-scale (see Barvainis 1987; Robson et al. 1993; Sokolov \& Marscher 2005, and references therein). Thus, the overall model for the continuum emission from 3C273 is complex. In order to investigate the underlying physics of the quasar, and particularly the origin of the X-ray emission, we must therefore adopt a method that can isolate the different components. Our approach is to study variability of the continuum emission in different wavebands, looking for correlations that reveal 
the primary emission mechanisms and their locations in the quasar (e.g. jet or Seyfert-like nucleus). In order to particularly search for a relationship between the Compton-scattered X-ray photons and possible synchrotron seed photons, we (M ${ }^{\mathrm{c}}$ Hardy et al. 1999, hereafter Paper I) carried out a programme of correlated near-IR ( $K$ band, $2.2 \mu \mathrm{m}$ ) and medium-energy X-ray (3-20 keV) observations of 3C273 in 1996/97. These observations revealed a strong correlation between the emission in the two bands and showed that the X-rays lag the IR by $0.75 \pm 0.25 \mathrm{~d}$.

These previous observations, although the first serious IR/X-ray variability observations of a blazar, were not extensive. One $\mathrm{X}$-ray flare was reasonably covered by IR observations and a second was sparsely covered. However, it was not clear whether these observations were representative of the general IR/X-ray behaviour of 3C273. It is possible that the IR and X-ray variations may not have been physically correlated and may have been merely chance events [e.g. see discussion in Mason et al. (2002)]. In order to clarify whether the previously observed IR/X-ray correlation was, indeed, representative of the general behaviour of $3 \mathrm{C} 273$, we therefore repeated the experiment in 1999 March, with better temporal coverage.

Here, we report the result of the 1999 March observations, during which a flare was seen first at $K$ band and then, with a delay of $1.45 \pm 0.15 \mathrm{~d}$, in X-rays. We discuss the combined significance of the 1996/97 and 1999 observations and consider various theoretical interpretations. We show that the most reasonable explanation of the X-ray variability is synchrotron self-Compton (SSC) scattering. In Section 2, we describe the observations, and in Section 3 we determine the lag between the X-ray and IR bands. In Section 4 we compare our observations with theoretical models, and in Section 5 we summarize our conclusions.

\section{OBSERVATIONS: SECOND CAMPAIGN}

\subsection{X-ray observations}

We observed 3C273 from 1999 March 1 to 15 with the Proportional Counter Array (PCA) on RXTE. The only significant difference from the 1996/97 observations is that only two of the Proportional Counter Units (PCUs) of the PCA were in operation, compared to three in the previous observations; hence the total count rate is down by 33 per cent relative to Paper I for the same photon flux. The data were reduced in exactly the same standard way as is described in Paper I, where the reader can find details of the analysis.

We observed the source for a total of $\sim 250 \mathrm{ksec}$, with approximately $5 \mathrm{~h}$ per day on target. The observation time per day was therefore much more than in the previous observations, although the $5 \mathrm{~h}$ were not usually spread evenly throughout the day but typically occurred in two or three blocks. After rejection of data that did not satisfy the standard acceptance criteria, a total of $172 \mathrm{ksec}$ of good-quality data were retained. X-ray variability on very short time-scales $(<1 \mathrm{ksec})$ is of low amplitude in $3 \mathrm{C} 273$, so these data were split into segments of $\sim 5 \mathrm{ksec}$ duration. In Fig. 1, we show the resultant 3-20 keV count rate light curve. As in Paper I, continuous $\mathrm{X}$-ray variability is seen on all time-scales longer than the bin size (5 ksec). The longer observation times compared to the 1996/97 observations result in smaller flux errors, which allows us to detect real variability at the few per cent level on time-scales of $\sim$ hours. As with Seyfert galaxies, 3C273 shows continual variations with larger amplitude variability on longer time-scales. There is a lack of flickering on very short time-scales (<hours) compared to Seyfert galaxies. This may be explained simply by a higher black hole mass, and therefore larger characteristic size scales, than in Seyfert galax- ies (e.g. McHardy et al. 2004; $M^{c}$ Hardy et al. 2005), but we have not yet completed a proper power-spectral analysis of the X-ray variability, and so are unable to make a quantitative comparison.

The observations in 1996/97 occurred when 3C273 was a little brighter, and the flares were of slightly larger amplitude, than in 1999 March. The mean level in Fig. 1 is approximately 75 per cent of the mean level shown in Paper I (reproduced here in Fig. 2). The X-ray flare beginning on 1999 March 3 has an amplitude of $\sim 25$ per cent of the mean level and is discernible above the mean level for about $4 \mathrm{~d}$, compared to a flare amplitude of $\sim 40$ per cent of the mean for the two flares in Paper I, each of which was discernible above the mean for about $10 \mathrm{~d}$.

\subsection{Near-IR observations}

$K$-band observations were made with the UFTI and IRCAM3 imaging cameras at United Kingdom Infrared Telescope (UKIRT) at the start, middle and end of each night from 1999 March 3 to 15, thereby improving temporal coverage by a factor of 3 compared to the 1997 observations. Typical exposures were of $3 \mathrm{~min}$ and were reduced in the standard manner, as described in Paper I. The resultant light curve is shown in Fig. 1. IRCAM $K$-band fluxes are transformed to the UFTI $K$ band by assuming a colour for 3C273 of $J-K=$ $2 \mathrm{mag}$, the value that we have measured in previous observations. Uncertainties in the transformation are included in the errors. Note that for the very first IR observation, the calibration star was 4 mag fainter than for all of the other observations, and so the error bars may be underestimated.

Additional $K$-band observations were made at the $0.75-\mathrm{m}$ telescope of the South African Astronomical Observatory (SAAO) during the UKIRT day in order to improve temporal coverage still further. Unfortunately, despite longer integration times, the resultant errors were factors of a few larger than the errors on the UKIRT observations, and so we do not include these data in the present analysis.

The large flare visible at the beginning of the X-ray light curve (Fig. 1) is also clearly seen in the IR light curve and appears to precede the X-ray light curve by about a day. After March 8, the X-ray and IR light curves display only 5-10 per cent variability, which does not appear to be strongly correlated between the bands, although we note that the dip in the X-ray light curve on March 12 would not have been sampled by the IR light curve had it occurred on March 11.

\section{X-RAY/INFRARED CROSS-CORRELATION}

We follow the same procedure as given in Paper I to determine the correlation between the X-ray and IR fluxes. We first perform a discrete cross-correlation (Edelson \& Krolik 1988) using all of the observations presented in Fig. 1. The result is given in Fig. 3, which shows that the X-rays lag the IR by approximately $1.5 \mathrm{~d}$.

In order to refine the measurement of the lag, we perform the same fitting operation that we used in Paper I. Assuming that the $\mathrm{X}$-rays result from scattering of the IR photons, we can parametrize the relationship by

$X_{\text {predicted }}(t)=A\left[K_{\text {flux }}(t-\delta t)-K_{\text {quiescent }}\right]^{N}+X_{\text {quiescent }}$.

$K_{\text {quiescent }}$ is a non-varying $K$-band component, probably from dust emission, of amplitude $50 \mathrm{mJy}$ (Robson et al. 1993). $K_{\text {flux }}(t-\delta t)$ is the total observed $K$-band flux at time $t-\delta t$, and $X_{\text {predicted }}(t)$ is then the predicted total X-ray flux at time $t$. $A$ is the constant of proportionality and $N$ contains information about the emission 


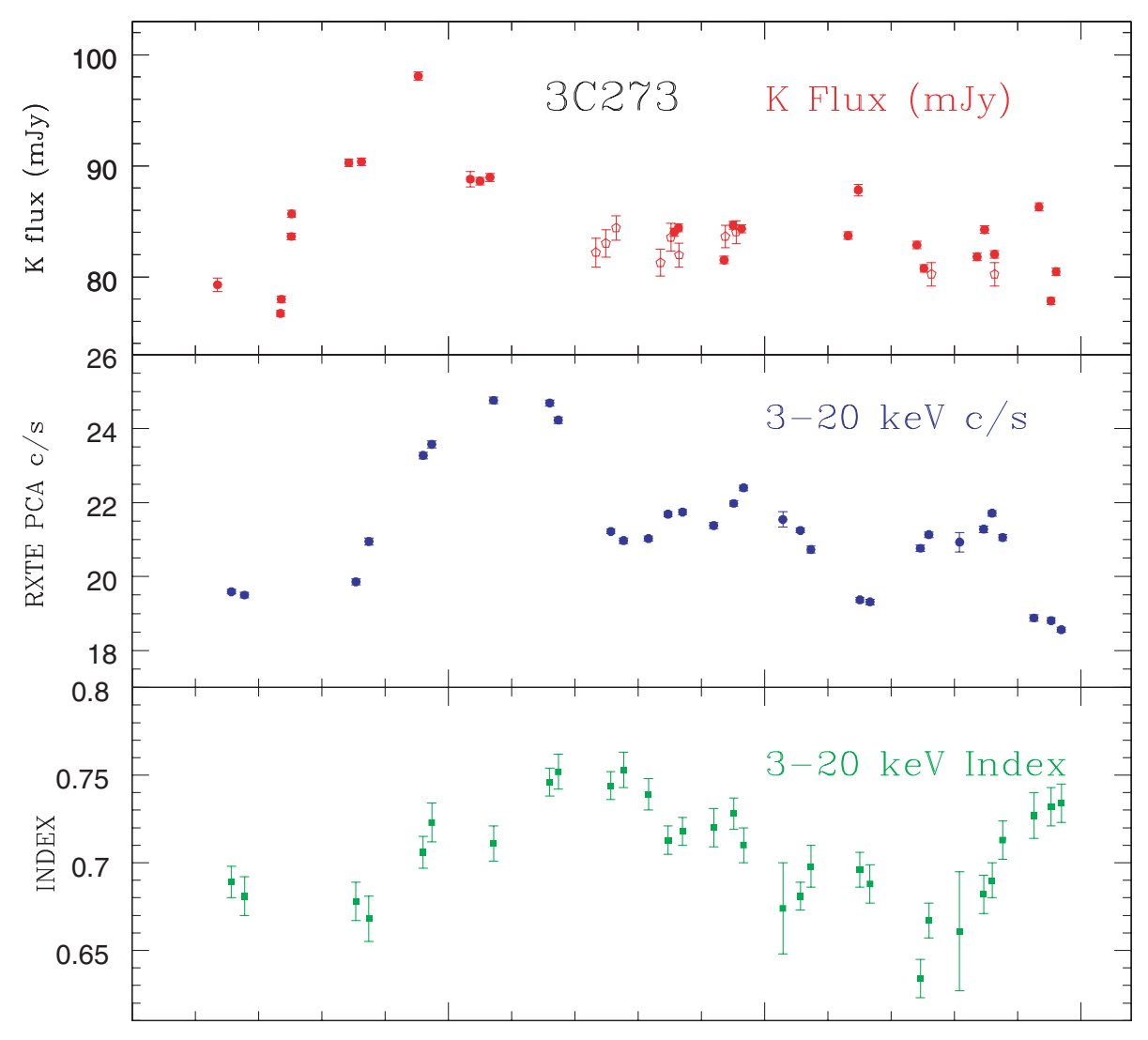

Days of March 1999 (UT)

Figure 1. X-ray and near-IR $K$-band light curves and X-ray spectral 'energy' index as a function of time. The X-ray counts are the total from 2 PCUs of the PCA. The time covered by each data point is between 4 and $8 \mathrm{ksec}$. Where definite variability is seen within an observation, two data points are given; otherwise only one is given. The error bars are plotted, but are generally too small to see. The $K$-band data from UKFTI on UKIRT are displayed as filled circles, the data from IRCAM3 on UKIRT are open pentagons. Note that the dates are labelled such that 0 March means 00:00 on 1999 March 1.

mechanism. For example, in the SSC process if the X-rays arise from variations in the number of high-energy electrons then we expect $N=2$, but $N=1$ if the variations result from changes solely in magnetic field strength.

$X_{\text {quiescent }}$ is the part of the X-ray flux that does not come from the flaring region. This emission could come from the Seyfert-like nucleus or from the 'quiescent' jet. Grandi \& Palumbo (2004) show that only $\sim 20$ per cent of the X-ray emission in 3C273 comes from the Seyfert-like nucleus. As the black hole in $3 \mathrm{C} 273$ is very large $\left(6 \times 10^{9} \mathrm{M}_{\odot} ;\right.$ Paltani \& Türler 2005), large amplitude variations of the Seyfert component on $\sim$ week time-scales will be unimportant (e.g. see $\mathrm{M}^{\mathrm{c}}$ Hardy et al. 2005) and, by definition, variations from the 'quiescent jet' will be on longer time-scales than those discussed here. Thus, although a slight variation of $X_{\text {quiescent }}$ may occur over the $\sim$ weeks time-scale of our observations, it is adequate for the purposes of examining only the larger, and more pronounced, variations to treat $X_{\text {quiescent }}$ as a constant. We have experimented with allowing $X_{\text {quiescent }}$ to vary with a linear trend, and the best fit is for a decrease, in the 1996/97 observations, of about 10 per cent over the observations. But the derived values of the other parameters do not change significantly and the improvement in fit is not sufficient to justify the inclusion of another free parameter.
The variable $\delta t$ allows for lags between the X-ray and IR variations. Neither the previous nor the present observation allows us to tightly constrain $N, A$ or $X_{\text {quiescent }}$, but varying $\delta t$, whilst allowing the other variables to remain free does affect the fit significantly. As in Paper I, we therefore perform a $\chi^{2}$ fit, comparing the predicted X-ray flux with the observed flux, using a standard Levenburg-Marquardt minimization routine, for various values of $\delta t$. We apply simple linear interpolation to estimate the X-ray flux at the exact (shifted) time of the IR observations. ${ }^{1}$ The results are shown in Fig. 4. In this fit, we use only the data prior to March 8 , covering the large flare. If we use all of the data, the fit becomes worse as it becomes dominated by the relatively quiescent levels after March 8, although it still indicates that the IR leads the X-rays.

The minimum $\chi^{2}$ value occurs when the IR leads the X-rays by $1.45 \mathrm{~d}$. The high absolute values of $\chi^{2}$ reflect the fact that the errors of both the X-ray and the IR fluxes are low and that, although the flare is broadly of the same shape in both bands, the detailed behaviour is not exactly the same. In other words, the model, although reasonable to

\footnotetext{
${ }^{1}$ In Paper I, we followed exactly the same procedure but the paper incorrectly states that we interpolated the IR flux to the exact (shifted) time of the X-ray observations. In any case, the results do not depend significantly on whether one interpolates the IR or the X-ray data.
} 


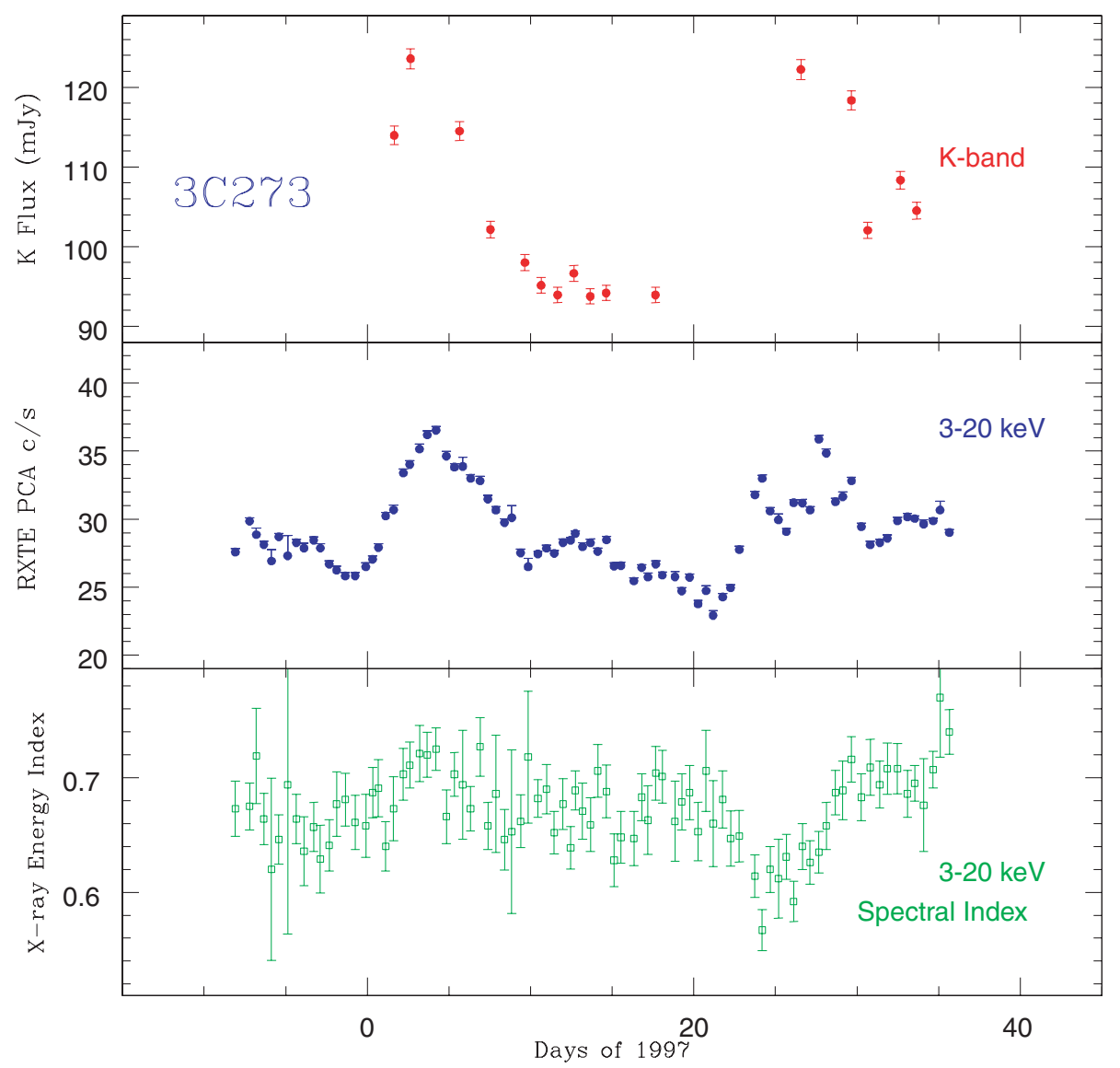

Figure 2. X-ray and near-IR $K$-band light curves and X-ray spectral 'energy' index as a function of time. The X-ray counts are normalized to 2 PCUs, for consistency with Fig. 1. The time covered by each X-ray data point is $1 \mathrm{ksec}$. The error bars are plotted, but are generally too small to see. Note that the dates are labelled such that 0 means 00:00 on 1997 January 1.

zeroth order, does not provide a detailed description of the observed behaviour. Therefore, we cannot obtain an error on the lag by simply measuring the difference in lag at some value of $\delta \chi^{2}$ away from the minimum which, for a good model fit, might give us a 90 per cent confidence region (see Press et al. 1992, section 15.6, for details). We have therefore carried out a Monte Carlo simulation, scattering all the data points by their errors and refitting. After 10000 such simulations, we derive an error of $0.05 \mathrm{~d}$ for the lag.

Using the parameters and lag defined by our best fit, we predict the X-ray flux and show it, superposed on the observed flux, in Fig. 5. As in Paper I, we see that the fit to the flare at the start of the observational period is good but once the flare has faded the correspondence becomes looser, presumably as additional minor components, for example the Seyfert-like nucleus, also contribute notably to the flux (see Grandi \& Palumbo 2004). Our sole interest here is to analyse the most pronounced flares, hence we will ignore the post-flare behaviour, our data for which are too limited to interpret.

\section{COMPARISON WITH THEORETICAL MODELS}

In both 1997 and 1999, we have observed reasonably well resolved flares in 3C273 where the X-ray emission lags the IR emission by about a day and, on both occasions, the X-ray spectrum of the flaring emission was softer than the quiescent emission. Staring around day
20 of 1997, we also note a second, more irregular period of enhanced $\mathrm{X}$-ray emission where the lag is probably shorter and where the $\mathrm{X}$-ray spectrum becomes harder during the enhanced emission. In this section, we consider the implications of these observations for theoretical models of its X-ray emission and for the specific structure of the jet and X-ray emission region in 3C273.

\subsection{Summary of X-ray emission mechanisms}

A perturbation, e.g. a shock or other compression, in the jet leading to higher electron density and enhanced synchrotron emission would lead to greater scattering of the ambient nuclear UV/optical/IR photon field, resulting in an increase in X-ray and Gamma-ray flux (the External Compton, or EC, model; Dermer \& Schlickeiser 1993; Sikora, Begelman \& Rees 1994; Błażejowski et al. 2000). However, the synchrotron photons must also be scattered by their parent electrons (SSC model; Jones, O'dell \& Stein 1974), producing an elevated level of high-energy emission. One of our main aims here is to distinguish which mechanism is more important for the nonthermal X-ray emission in 3C273.

A previously popular variant of the SSC model was the Mirror Compton (MC) model (Ghisellini \& Madau 1996). Here, the synchrotron photons from the jet are reflected back to the jet from a neighbouring cloud with the scattered emission lagging the synchrotron emission by approximately the light travel distance to the cloud. However, detailed calculation shows that any reflecting cloud 


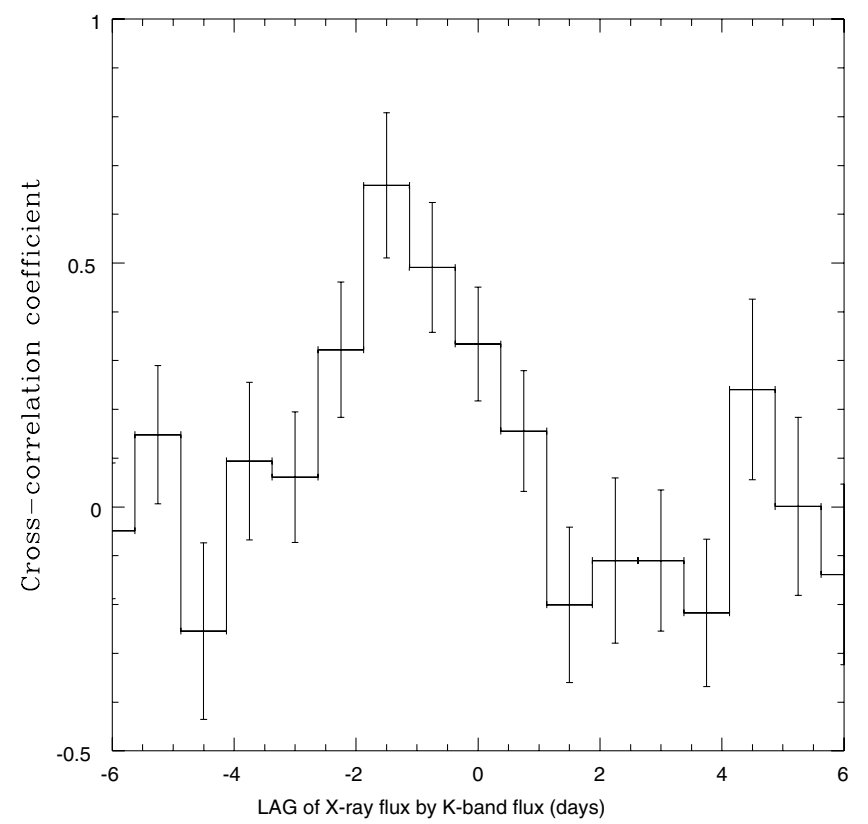

Figure 3. Cross-correlation of the X-ray and IR fluxes. The bin size is $0.75 \mathrm{~d}$.

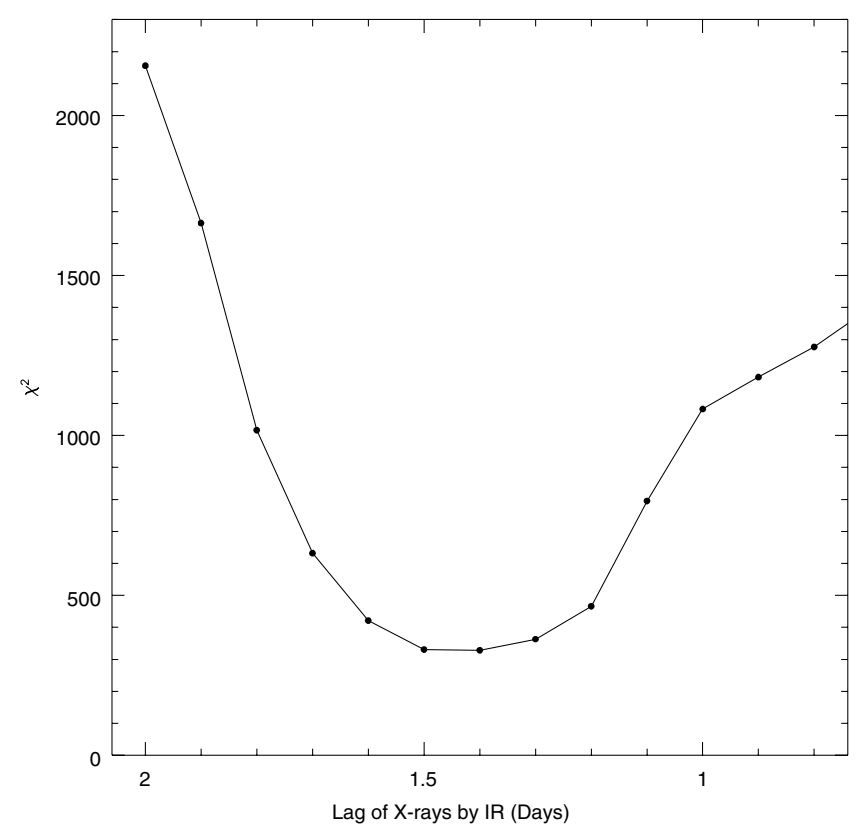

Figure 4. Result (in terms of the $\chi^{2}$ statistic) of comparing the observed $\mathrm{X}$-ray light curve with that predicted from the IR variations as a function of lag. For each value of the lag, all other parameters are allowed to remain free.

would need to lie essentially in the jet to produce a significant scattered high-energy flare (Bednarek 1998; Boettcher \& Dermer 1998). Thus, the MC model is unlikely to be the cause of the majority of $\mathrm{X}$-ray flares.

\section{2 $\mathrm{X}$-ray/IR lags as emission diagnostics}

There are a number of variables, e.g. the geometry of the emitting region and the angle of the jet to the line of sight, which can affect the decay time-scales, amplitudes and lag between the X-ray and

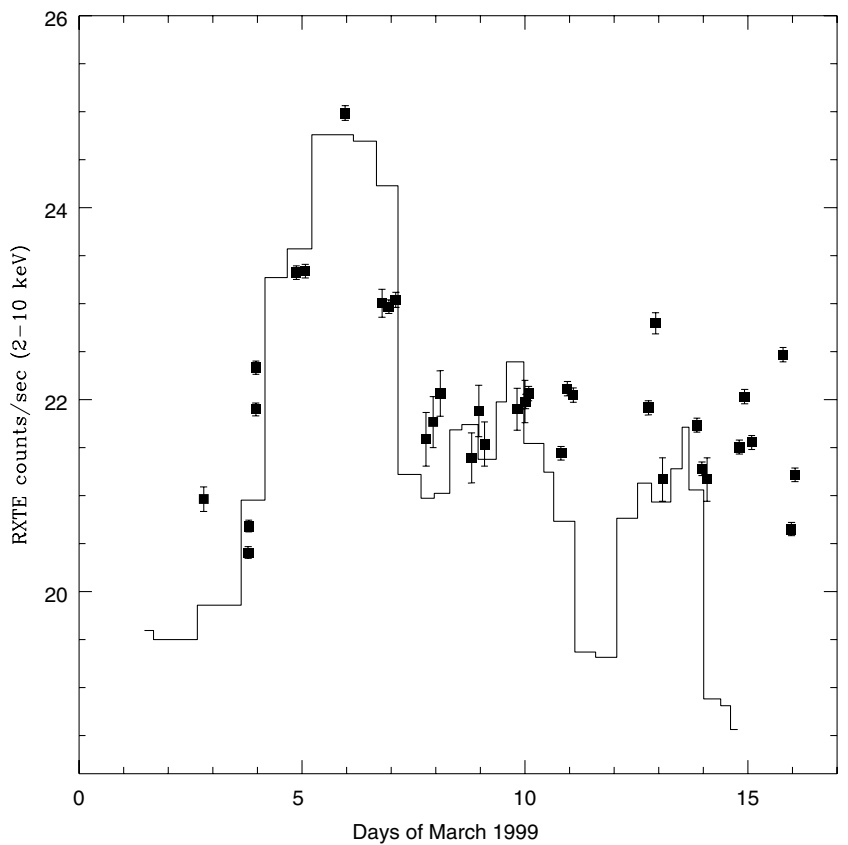

Figure 5. Observed X-ray light curve (histogram) and the best-fitting predicted X-ray flux (filled squares) based on the parameters derived from fitting the IR observations to the initial flare (20 IR data points) only. The best-fitting parameters are $A=0.24, N=0.98$ and $X_{\text {quiescent }}=14.5$. Following from Fig. 4 , the lead of the IR over the observed X-rays is fixed at $1.45 \mathrm{~d}$, the bestfitting value for the first flare. The observed X-ray error bars (see Fig. 1) are not repeated here to avoid cluttering the diagram.

IR emission. We refer to Sokolov, Marscher \& $\mathrm{M}^{\mathrm{c}} \mathrm{Hardy}$ (2004) for a general discussion on the modelling of non-thermal flares similar to those shown here. Here, at the risk of some oversimplification, we concentrate on the two general points of most relevance to observations.

First, in the EC process, the electrons responsible for synchrotron IR flares have high energies $(\gamma \sim 1000)$ and so decay quickly, whereas the electrons responsible for scattering IR/optical/UV photons to $\mathrm{X}$-ray energies are of low energy $(\gamma \sim 10)$ and so have much longer decay time-scales. Thus, the X-ray decay time-scale is much longer than the IR decay time-scale and so we do not expect flares to look similar in both bands. However, in the SSC process, higher energy electrons contribute strongly to the X-ray emission (see e.g. Paper I) and so we expect the X-ray and IR flares to have moderately similar shapes and be better correlated than in the EC case. The similarity of our observed X-ray and IR flares therefore favours the SSC model.

Secondly, in the EC process, the synchrotron and X-ray variations should rise almost simultaneously as the ambient UV/optical/IR field is all pervasive and is immediately available for scattering to $\mathrm{X}$-ray energies. However, in the SSC process there are two reasons why high-energy flares might lag IR or optical synchrotron flares. If the jet points almost along the line of sight, then the seed photon field does not rise instantaneously at the location of any given electron, but reaches a peak after approximately half of the light travel time across the emission region. The X-ray emission should therefore lag the synchrotron emission by about the same time. The more general reason for a lag is frequency stratification within the source (Marscher \& Gear 1985). The SSC flux at any given Xray energy is produced by a combination of electron energies and seed photon frequencies, including relatively low-energy electrons 
scattering high-frequency photons to high-energy electrons scattering low-frequency photons (see fig. 3 in Paper I). If the higher energy electrons and high-frequency synchrotron photons are confined to a small volume, e.g. close to a shock front, the X-ray flare, which comes from a more extended region, will have a time-delayed maximum (and may also be more prolonged than the highest frequencies in the synchrotron flare). The delay of the X-ray relative to the IR therefore again favours the SSC over the EC model for the origin of the $\mathrm{X}$-ray emission.

The $2-10 \mathrm{keV}$ emission arises from self-Compton scattering of a large range of seed photon energies, from radio through to IR, by a large range of electron energies (see Paper 1). However, high-energy Gamma-ray emission is produced by scattering, by the highest energy electrons, of the higher energy seed photons (optical/UV). The Gamma-ray emission therefore need not necessarily be dominated by SSC emission but could arise, at the higher $(\mathrm{GeV})$ energies, from scattering of ambient optical/UV photons from the accretion disc and broad line region (Kubo et al. 1998; Dermer \& Schlickeiser 1993; Sikora et al. 1994) or, at MeV energies, even by scattering of IR emission from the torus (Błażejowski et al. 2000).

\subsection{The basic jet structure}

The short-time-scale near-IR flares are almost certainly enhancements of the synchrotron emission from the jet. The time-scale of variability of $\sim 2 \mathrm{~d}$ implies that the flaring region has a maximum size of $\sim 2 \delta \sim 20$ lt-days $\sim 0.02 \mathrm{pc}$, where the value of the relativistic Doppler factor, $\delta \approx 9$ (Lorentz factor 11 , angle to the line of sight $6^{\circ}$ ) in the jet in early 1999 was derived by Jorstad et al. (2005) from apparent superluminal motion and the decay time of the flux of moving radio knots. These authors also determined the opening half-angle of the jet to be $1: 4 \pm 0.3$. If the jet has a conical geometry, the distance from the apex corresponding to $0.02 \mathrm{pc}$ is $\sim 0.5 \mathrm{pc}$, or greater if the flaring region does not span the entire width of the jet.

\subsection{X-ray spectral variability: the emission region}

Precise interpretation of X-ray spectral variability is difficult as we are almost certainly seeing the superposition of a number of different events, so we restrict ourselves to commenting only on the larger events.

In the 1999 March flare (Fig. 1) and in the first of the 1997 January flares (Fig. 2), we note broadly similar spectral variability. In each case, the spectrum of the flare is softer than that of the quiescent state, which is most simply explained if the injected electron energy distribution is steeper than that of the quiescent jet. Such an interpretation is consistent with the observation that the X-ray spectral index returned to the previous value as the flare died out. We do note, in the better sampled 1999 March flare, that the peak in the spectral index lags that in X-ray flux by about a day or so, probably indicating ageing of the flare electron distribution caused by radiative losses.

However, in the second 1997 flare we note the reverse behaviour as the spectrum initially hardens with increasing flux before softening back towards the quiescent value. This spectral difference may be related to temporal differences. Unlike the smooth first flare, the second flare appears to be made up of three or four smaller flares, superposed, and the rise of the second flare is more abrupt than that of the first flare. Also, although not as well constrained by observation, we noted in Paper I that the $\sim 1 \mathrm{~d}$ lag of the IR by the X-rays during the first flare did not easily apply to the second flare, and that a zero delay was a better fit. A possible explanation, therefore, is that in the second 1997 flare we see not just one instance of excitation (e.g. a shock), but a succession of small regions of particle acceleration, perhaps with one shock compounding the energization of the previous one (e.g. as in a colliding shock model; Spada et al. 2001). Thus, the dominant emission region remains small, consistent with the very small IR-X-ray lag, and particles are continually reaccelerated, a situation that favours the acceleration of electrons to high rather than low energies. In this scenario, the X-ray spectral variability reflects the multiplicity of episodes of re-energization of the jet plasma. The observed spectral differences in the flares shown here may therefore simply be reflecting the spectrum of turbulence in the underlying jet.

\section{CONCLUSIONS}

Our present observations repeat the general pattern of X-ray and IR variability described in Paper I. X-ray variability in 3C273 is seen on all time-scales which are currently available to observation, i.e. > hours. We again demonstrate that there is a strong correlation between variations in the X-ray and IR bands for variations of amplitude $>20$ per cent, confirming that the observations described in Paper I were not the result of chance coincidence. For lower amplitude variations the correlation is less clear, probably indicating that multiple emission regions and processes are involved (see e.g. Courvoisier 1998; Grandi \& Palumbo 2004).

The observations presented here and in Paper I were not scheduled at any particularly special time, or flux level, and so confirm that the behaviour presented here, and in Paper I, is typical of 3C273. In other words, the same mechanism dominates the X-ray emission of $3 \mathrm{C} 273$ during small flares, and the structure of the emission region during those flares must broadly be the same. Slight differences in jet properties, e.g. location and size of the flaring region, shock strength, bulk Lorentz factor and direction of the jet, can easily alter the flare amplitude and lag time-scale. We note that the latter changed only from $0.75 \pm 0.25$ to $1.45 \pm 0.15 \mathrm{~d}$, a quite modest difference given the number of variables involved.

We note that the profile of temporal variability is linked to how the $\mathrm{X}$-ray spectrum varies and provides a good indicator of the structure of the underlying emission region.

The fact that there is again a strong correlation between the $\mathrm{X}$-ray and IR variations, with flares of broadly similar shapes with the X-ray maximum lagging the IR peak, strongly supports the SSC, rather than EC, model for the production of the X-rays in 3C273. As in Paper I, our simplistic fitting procedure does not significantly constrain the parameters $A, N$ or $X_{\text {quiescent }}$ although, as in Paper I, values of $N$ nearer to 1 than 2 are preferred indicating, in the SSC model, that variations in magnetic field strength as well as electron density are important in the X-ray variations.

\section{ACKNOWLEDGMENTS}

We thank the management and operational staff of both $R X T E$ and UKIRT for their cooperation in scheduling and carrying out these observations. IM thanks PPARC for support by grant GRK21931 and by the award of a Senior Fellowship. The Boston University effort was supported in part by the National Science Foundation through grants AST-0098579 and AST-0406865 and by NASA through grants NAG5-11811 and NAG5-13074.

\section{REFERENCES}

Barvainis R., 1987, ApJ, 320, 537

Bednarek W., 1998, A\&A, 336, 123 
Błażejowski M., Sikora M., Moderski R., Madejski G. M., 2000, ApJ, 545, 107

Boettcher M., Dermer C. D., 1998, ApJ, 501, L51

Courvoisier T. J.-L., 1998, A\&AR, 9, 1

Courvoisier T. J.-L. et al., 2003, A\&A, 411, L343

Dermer C. D., Schlickeiser R., 1993, ApJ, 416, 458

Edelson R. A., Krolik J. H., 1988, ApJ, 333, 646

Ghisellini G., Madau P., 1996, MNRAS, 280, 67

Grandi P., Palumbo G. G. C., 2004, Sci, 306, 998

Jones T. W., O'dell S. L., Stein W. A., 1974, ApJ, 188, 353

Jorstad S. G., Marscher A. P., Mattox J. R., Wehrle A. E., Bloom S. D., Yurchenko A. V., 2001, ApJS, 134, 181

Jorstad S. G. et al., 2005, AJ, 130, 1418

Kubo H., Takahashi T., Madejski G., Tashiro M., Makino F., Inoue S., Takahara F., 1998, ApJ, 504, 693

Mantovani F., Junor W., Valerio C., McHardy I., 1999, A\&A, 346, 397

Marscher A. P., Gear W. K., 1985, ApJ, 298, 114

Mason K. O. et al., 2002, ApJ, 580, L117

Mㄷardy I., Lawson A., Newsam A., Marscher A., Robson I., Stevens J., 1999, MNRAS, 310, 571 (Paper I)
McHardy I. M., Gunn K. F., Uttley P., Goad M. R., 2005, MNRAS, 359, 1469

M ${ }^{\mathrm{c} H a r d y ~ I . ~ M ., ~ P a p a d a k i s ~ I . ~ E ., ~ U t t l e y ~ P ., ~ P a g e ~ M . ~ J ., ~ M a s o n ~ K . ~ O ., ~ 2004, ~}$ MNRAS, 348, 783

Paltani S., Türler M., 2005, A\&A, 435, 811

Press W. H., Teukolsky S. A., Vetterling W. T., Flannery B. P., 1992, Numerical Recipes in FORTRAN. The Art of Scientific Computing, 2nd edn. Cambridge Univ. Press, Cambridge

Robson E. I., Gear W. K., Brown L. M. J., Courvoisier T. J.-L., Smith M. G., 1986, Nat, 323, 134

Robson E. I. et al., 1993, MNRAS, 262, 249

Sikora M., Begelman M. C., Rees M. J., 1994, ApJ, 421, 153

Sokolov A., Marscher A. P., 2005, ApJ, 629, 52

Sokolov A., Marscher A. P., McHardy I. M., 2004, ApJ, 613, 725

Spada M., Ghisellini G., Lazzati D., Celotti A., 2001, MNRAS, 325, 1559

Valtaoja E., Valtaoja L., Efimov I. S., Shakhovskoi N. M., 1990, AJ, 99, 769

This paper has been typeset from a $\mathrm{T}_{\mathrm{E}} \mathrm{X} / \mathrm{LAT}_{\mathrm{E}} \mathrm{X}$ file prepared by the author. 